\title{
非均相芬顿体系协同去除复合双污染物：化学转化, $\mathrm{pH}$ 影响和 机理分析
}

\author{
杨波张永丽* \\ (四川大学建筑与环境学院 成都 610065)
}

\begin{abstract}
摘要 系统研究了 ZVI(零价铁粉)-Fenton 体系协同去除铜离子和亚甲基蓝(MB)污染物过程中, ZVI 微表面发生的化学 转化以及目标污染物降解机理. 分别利用扫描电子显微镜( SEM), X 射线能谱(EDS), $X$ 射线衍射(XRD), $X$ 射线光电子能 谱(XPS)和傅里叶变换红外光谱(FTIR)等技术, 对比分析了反应前后以及不同体系之间 ZVI 表面结构, $\mathrm{Fe}$ 和 $\mathrm{Cu}$ 化学转 移的变化. 结果表明, 在 $\mathrm{ZVI} / \mathrm{H}_{2} \mathrm{O}_{2}$ 体系中反应后 $\mathrm{ZVI}$ 表面腐蚀产物较多, 主要为 $\mathrm{Fe}_{3} \mathrm{O}_{4}$ 和 $\mathrm{Fe}_{2} \mathrm{O}_{3}$. 在 $\mathrm{ZVI} / \mathrm{H}_{2} \mathrm{O}_{2}-\mathrm{Cu}$ 体系 中, 虽 ZVI 腐蚀作用更加剧烈, 但 ZVI 表面残留的腐蚀产物较少, 且腐蚀产物中 $\mathrm{Fe}_{3} \mathrm{O}_{4}$ 含量的占比增加. $\mathrm{Cu}^{2+}$ 主要还原 产物为 $\mathrm{Cu}^{0}$, 同时还伴随着 $\mathrm{CuO}$ 的生成. $\mathrm{pH}$ 影响实验表明, $\mathrm{ZVI} / \mathrm{H}_{2} \mathrm{O}_{2}-\mathrm{Cu}$ 体系不仅强化了 $\mathrm{MB}$ 的降解, 有效地去除了总 溶解铜离子 $(\mathrm{TCu})$, 同时还扩大 ZVI-Fenton 体系的有效 $\mathrm{pH}$ 范围 $(\mathrm{pH}=2.5 \sim 5.5)$. 叔丁醇捕获自由基实验表明, 羊基自由 基是氧化降解 MB 的主要活性物质. 最后针对 ZVI-Fenton 体系协同去除复合双目标污染物的机理进行研究分析.

关键词 零价铁粉; 非均相芬顿; 循环催化; 协同作用; 微表面转化
\end{abstract}

\section{Synergistic Removal of Co-contamination by Heterogeneous Fenton System: Chemical Conversion, pH Effect and Mechanism Analysis}

\author{
Yang, Bo Zhang, Yongli* \\ (College of Architecture \& Environment, Sichuan University, Chengdu 610065, China)
}

\begin{abstract}
The chemical transformation of ZVI micro-surface and the degradation mechanism in the process of synergistic removal of copper ions and methylene blue pollutants by ZVI-Fenton system were studied systematically. The samples of $\mathrm{ZVI}$, before and after reaction in the $\mathrm{ZVI} / \mathrm{H}_{2} \mathrm{O}_{2}$ and $\mathrm{ZVI} / \mathrm{H}_{2} \mathrm{O}_{2}-\mathrm{Cu}$ systems, were characterized by scanning electron microscopy (SEM), energy dispersive X-ray spectrometer (EDS), X-ray diffraction (XRD), X-ray photoelectron spectra (XPS) and Fourier Transform infrared spectroscopy (FTIR) to research the changes of ZVI surface structure, $\mathrm{Fe}$ and $\mathrm{Cu}$ species' chemical conversion. The results showed that the residual corrosion products on the surface of ZVI were more and the corrosion products were mainly $\mathrm{Fe}_{3} \mathrm{O}_{4}$ and $\mathrm{Fe}_{2} \mathrm{O}_{3}$ after reaction in the $\mathrm{ZVI} / \mathrm{H}_{2} \mathrm{O}_{2}$ system. However, in the $\mathrm{ZVI} / \mathrm{H}_{2} \mathrm{O}_{2}-\mathrm{Cu}$ system, the corrosion effect of ZVI was more significant, but the residual corrosion products of ZVI surface were less, and the proportion of $\mathrm{Fe}_{3} \mathrm{O}_{4}$ increased. In addition, the main reduction product of $\mathrm{Cu}^{2+}$ was $\mathrm{Cu}^{0}$, which was accompanied by the generation of $\mathrm{CuO}$. Furthermore, the effects of $\mathrm{pH}$ on the removal of pollutants from the five systems ( $\mathrm{ZVI}, \mathrm{ZVI}-\mathrm{Cu}, \mathrm{H}_{2} \mathrm{O}_{2}-\mathrm{Cu}, \mathrm{ZVI} / \mathrm{H}_{2} \mathrm{O}_{2}$ and $\mathrm{ZVI} / \mathrm{H}_{2} \mathrm{O}_{2}-\mathrm{Cu}$ ) were compared and the changes in $\mathrm{TCu}$ and TFe concentrations under different $\mathrm{pH}$ conditions were monitored. The results indicated that the $\mathrm{ZVI} / \mathrm{H}_{2} \mathrm{O}_{2}-\mathrm{Cu}$ system not only simultaneous effectively remove $\mathrm{MB}$ and $\mathrm{TCu}$ compared with other three systems, but also enlarged the effective $\mathrm{pH}$ range $(\mathrm{pH}=2.5 \sim 5.5)$ of ZVI-Fenton system. In addition, free radical capture experiments showed that hydroxyl radicals played an important role in the oxidative degradation of methylene blue, and $10 \mathrm{mmol} / \mathrm{L}$ tert-butanol could completely capture hydroxyl radicals in the system. Finally, the mechanism of synergistic removal of $\mathrm{TCu}$ and $\mathrm{MB}$ degradation by ZVI-Fenton system was revealed. The substitution reaction between ZVI and $\mathrm{Cu}^{2+}$, the action of $\mathrm{Cu}^{0}$ and ZVI galvanic cells, the acid corrosion effect, and the redox cycle of iron and copper together accelerate the degradation of MB by the system and promote the conversion of ZVI surface substances. This study provides a theoretical basis for collaborative treatment of industrial complex pollutants.
\end{abstract}

Keywords zero-valent iron powder; heterogeneous Fenton; cyclic catalysis; synergism; micro surface transformation

\section{1 引言}

近年来, 零价铁粉(ZVI)因为具有廉价易得、绿色无 毒等优点 ${ }^{[1]}$, 同时还具有：还原降解作用、氧化降解作
用、化学沉淀作用和吸附作用等四个方面的作用 ${ }^{[2]}$, 被 广泛运用于环境治理研究中. 有关 ZVI 催化氧化剂(过 硫酸盐 ${ }^{[3]}$ ，双氧水 ${ }^{[4]}$ 等)，产生具有强氧化性的自由基 $\left(\cdot \mathrm{SO}_{4}^{-}, \cdot \mathrm{OH}\right)$ 降解有机污染物的研究一直持续不断, 其

\footnotetext{
*E-mail: zxm581212@163.com; Tel.: 028-85408889

Received June 8, 2019; published September 4, 2019.

Project supported by the National Natural Science Foundation of China (No. 51878422).

项目受国家自然科学基金(No. 51878422)资助.
} 
中 ZVI 与双氧水 $\left(\mathrm{H}_{2} \mathrm{O}_{2}\right)$ 结合的异相芬顿(ZVI-Fenton)又 是高级氧化技术方面的研究热点. 因为 ZVI-Fenton 体系 中 ZVI 代替传统均相芬顿 $\left(\mathrm{Fe}^{2+}\right.$-Fenton)体系中 $\mathrm{Fe}^{2+}$ 作为 持续稳定的铁源, 能有效地解决操作 $\mathrm{pH}$ 低、反应速率 无法有效控制以及含铁污泥造成二次污染等缺点 ${ }^{[5,6]}$. 但是 ZVI-Fenton 也存在瓶颈问题, 由于 ZVI 比表面积 大, 表面容易被氧化形成氧化层, 从而抑制 $\mathrm{Fe}^{2+}$ 从 ZVI 表面释放, 导致 ZVI-Fenton 体系催化活性降低. 因此很 多研究通过 ZVI 与过渡金属合成双金属 $\left(\mathrm{Fe} / \mathrm{Cu}^{2}\right.$, $\left.\mathrm{Fe} / \mathrm{Pd}^{[7]}, \mathrm{Ag} / \mathrm{Fe}^{[8,9]}, \mathrm{Fe} / \mathrm{Ni}^{[10]}, \mathrm{Fe} / \mathrm{Co}^{[11]} . . . ..\right)$, 通过原电池 效应来促进 ZVI 的腐蚀作用, 从而改善 ZVI 催化活性. 但是由于合成双金属的操作困难和成本较高 ${ }^{[12]}$, 且过 渡金属大部分是有剧毒的, 无法避免在反应过程中过渡 金属溢出造成二次污染 ${ }^{[13]}$. 因此, 探索绿色高效的技术 提高 ZVI-Fenton 的氧化活性一直以来都是研究热点.

此外, 因为 ZVI 的标准氧化还原电势低 $\left(E^{0}=-0.44\right.$

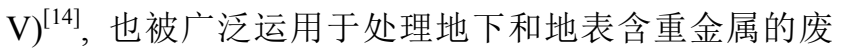
水 ${ }^{[1]}$. ZVI 处理重金属主要是通过置换作用实现电子转 移, 将毒性较大的高价金属离子转化为毒性较小的或者 无毒的低价金属. 还原后的金属附着在 ZVI 表面或者发 生沉积从而实现重金属的去除 ${ }^{[15]}$. Jiang 等 ${ }^{[16]}$ 研究了 ZVI 处理含 $\mathrm{Cu}^{2+}$ 重金属废水, 在弱磁场下提高了 $\mathrm{ZVI}$ 还原 $\mathrm{Cu}^{2+}$ 的反应速率. $\mathrm{Fu}$ 等 ${ }^{[17]}$ 报道称在 $\mathrm{pH}=6$ 条件下采用海 泡石负载 nZVI 能处理铬(VI)和铅(II)的去除率高到 $95 \%$ 以上. 但是 ZVI 与重金属的置换作用还原重金属的同时 伴随着大量的 $\mathrm{Fe}^{2+}$ 从表面释放, 且释放的 $\mathrm{Fe}^{2+}$ 并未得到 有效的利用.

工业废水往往含有多种污染物, 其中重金属和有机 污染物是两大类主要的污染物. 然而, 使用 ZVI 同时处 理含有重金属和有机污染物的工业废水的研究还比较 少. Diao 等 ${ }^{[18]}$ 通过结合 ZVI 和光催化剂 $\mathrm{TiO}_{2}$ 处理含重金 属六价铬和抗生素环丙沙星; Liu 等 ${ }^{[19]}$ 通过生物活性炭 负载分散 ZVI 激活过硫酸盐处理含 $\mathrm{Cu}^{2+}$ 和有机污染物 双酚 A. 但是光催化工艺存在光透射效率低, 且活性炭 负载 ZVI 的材料制备复杂等缺点. 基于以上分析, 使用 ZVI-Fenton 直接处理含有重金属和有机污染物的工业 废水, 不仅能解决 ZVI-Fenton 体系在单独处理有机污染 物时存在 $\mathrm{Fe}^{2+}$ 释放受到抑制, 催化活性低等问题, 同时 还能有效利用 ZVI 还原重金属过程中释放的 $\mathrm{Fe}^{2+}$.

本研究选取 $\mathrm{Cu}^{2+}$ 和亚甲基蓝(MB)作为目标污染物, 深入研究 ZVI-Fenton 体系 $\left(\mathrm{ZVI} / \mathrm{H}_{2} \mathrm{O}_{2}-\mathrm{Cu}\right)$ 协同去除总溶 解铜离子 $(\mathrm{TCu})$ 同时强化 $\mathrm{MB}$ 降解的机理. 同时通过 SEM, EDS, XRD, XPS 和 FTIR 等表征技术，系统分析了 反应前后以及不同体系下 ZVI 的腐蚀程度、腐蚀产物以 及 $\mathrm{TCu}$ 还原产物. 此外, 还通过自由基捕获实验确定体 系中主要活性物质, 对比研究了在不同 $\mathrm{pH}$ 范围条件下 不同体系对 $\mathrm{MB}$ 和 $\mathrm{TCu}$ 去除作用以及 $\mathrm{Fe}$ 离子溶出浓度 的关系.

\section{2 实验结果与讨论}

\subsection{SEM 和 EDS 表征}

采用扫描电镜(SEM)和 $X$ 射线能谱(EDS)表征不同 体系反应前后的 ZVI 的表面形态和元素组成, 结果如图 1 所示. 图 1(a), (b)为反应前的 ZVI 的 SEM 和 EDS 图像, 反应前的 ZVI 表面光滑无明显腐蚀现象. 但是 ZVI 表面 $\mathrm{Fe}$ 元素占比为 $68.37 \%$, 氧元素占比为 $28.34 \%$ ，也表明 了 ZVI 表面存在薄薄的氧化层. 在 $\mathrm{ZVI} / \mathrm{H}_{2} \mathrm{O}_{2}$ 体系中反 应后的 ZVI 表面出现了明显的腐蚀(图 1(c), (d)), 相应 $\mathrm{Fe}$ 元素占比下降, 氧元素占比上升, 表面附着了较多结 构稀疏的铁氧化腐蚀产物. 根据 Sleiman 等 ${ }^{[20]}$ 的研究, 氧化产物可能为 $\mathrm{Fe}_{3} \mathrm{O}_{4}$ 和 $\mathrm{Fe}_{2} \mathrm{O}_{3}$. 图 1(e), (f)为 ZVI 在 $\mathrm{ZVI} / \mathrm{H}_{2} \mathrm{O}_{2}-\mathrm{Cu}$ 体系反应后的表面形态和元素组成图. $\mathrm{Fe}$ 元素的占比仅有 $36.06 \%$ ，同时有 $18.83 \%$ 的 $\mathrm{Cu}$ 元素的检 出. 此外 ZVI 表面出现了明显的裂痕, 但是附着在表面 腐蚀产物相比于 $\mathrm{ZVI} / \mathrm{H}_{2} \mathrm{O}_{2}$ 体系较少. 图 1(d), (f) EDS 图 谱中均出现了少量的 $\mathrm{S}$ 元素, 是 $\mathrm{SO}_{4}^{2-}$ 离子残留在 $\mathrm{ZVI}$ 表面. 此外, 反应过程中 $\mathrm{ZVI}$ 还能将少量的 $\mathrm{SO}_{4}^{2-}$ 离子 还原成 $\mathrm{S}$ 单质附着在 ZVI 表面.

\section{$2.2 \mathrm{XRD}$ 表征}

对反应前后的 ZVI 进行 X 射线衍射分析(XRD), 确 定反应前后 ZVI 表面晶相结构变化, 进一步确定反应过 程中 ZVI 表面氧化腐蚀产物. 由图 2 可以看出, 反应前 的 ZVI 分别在 $2 \theta=44.67^{\circ}, 65.02^{\circ}$ 和 $82.33^{\circ}$ 出现较强的特 征衍射峰，完全符合 $\mathrm{Fe}^{0}(\mathrm{JCPDS}$ 06-0696). 经过 $\mathrm{ZVI} / \mathrm{H}_{2} \mathrm{O}_{2}$ 体系反应后的 $\mathrm{ZVI}$ 的 $\mathrm{Fe}^{0}$ 特征衍射峰强度明显 降低, 同时伴随着磁铁矿 $\mathrm{Fe}_{3} \mathrm{O}_{4}$ (JCPDS 19-0629, $2 \theta=$ $30.10^{\circ}, 35.42^{\circ}, 43.05^{\circ}, 56.94^{\circ}$ 和 $\left.62.52^{\circ}\right)$ 和磁赤铁矿 $\mathrm{Fe}_{2} \mathrm{O}_{3}\left(\mathrm{JCPDS} 39-1346,2 \theta=30.24^{\circ}, 35.63^{\circ}\right.$ 和 $62.93^{\circ}$ )特征 衍射峰出现 ${ }^{[2,21]}$. 相比于 $\mathrm{ZVI} / \mathrm{H}_{2} \mathrm{O}_{2}$ 体系, $\mathrm{ZVI} / \mathrm{H}_{2} \mathrm{O}_{2}-\mathrm{Cu}$ 体 系中 $\mathrm{Fe}_{3} \mathrm{O}_{4} / \mathrm{Fe}_{2} \mathrm{O}_{3}$ 的特征衍射峰较弱, 仅仅在 $35.42^{\circ}$ (或 者 $35.63^{\circ}$ ) 出现了衍射峰. 结果表明, 相比于 $\mathrm{ZVI} / \mathrm{H}_{2} \mathrm{O}_{2}-\mathrm{Cu}$ 体系, $\mathrm{ZVI}$ 在 $\mathrm{ZVI} / \mathrm{H}_{2} \mathrm{O}_{2}$ 体系中反应后, 生 成的表面腐蚀产物更多, 且主要为 $\mathrm{Fe}_{3} \mathrm{O}_{4}$ 和 $\mathrm{Fe}_{2} \mathrm{O}_{3}$, 验证 了 SEM 图像的观察结果. 由于 $\mathrm{Fe}_{3} \mathrm{O}_{4}$ 和 $\mathrm{Fe}_{2} \mathrm{O}_{3}$ 两种物质 的衍射峰存在部分重合, 所以 $\mathrm{ZVI} / \mathrm{H}_{2} \mathrm{O}_{2}-\mathrm{Cu}$ 体系的腐蚀 产物还需通过 XPS 进一步分析.

\subsection{XPS 表征}

$X$ 射线光电子能谱技术(XPS)被用来表征 ZVI 表面 的元素价态, 分析确定 $\mathrm{Fe}$ 和 $\mathrm{Cu}$ 反应后产物, 结果如图 3 和表 1 所示. 图 3(a)为 XPS 总谱图, 可以看出, Fe $2 p$ 峰相比于反应前的 ZVI 增强较大, 同时在 $\mathrm{ZVI} / \mathrm{H}_{2} \mathrm{O}_{2}-\mathrm{Cu}$ 体系中, $\mathrm{Cu} 2 \mathrm{p}$ 峰被检测出来. 图 3(b), (d)分别为 $\mathrm{ZVI} / \mathrm{H}_{2} \mathrm{O}_{2}$ 和 $\mathrm{ZVI} / \mathrm{H}_{2} \mathrm{O}_{2}-\mathrm{Cu}$ 体系中 $\mathrm{Fe} 2 \mathrm{p}$ 的特征峰, 724.1 $\mathrm{eV}$ 和 $712.1 \mathrm{eV}$ 处的两个特征峰分别与 $\mathrm{Fe}_{2} \mathrm{O}_{3}$ 的 $2 \mathrm{p}_{1 / 2}$ 和 $2 \mathrm{p}_{3 / 2}$ 匹配 ${ }^{[22,23]}, 716.7 \mathrm{eV}$ 为 $\mathrm{Fe}_{2} \mathrm{O}_{3}$ 的卫星峰 ${ }^{[16,24]}$, 而 $710.2 \mathrm{eV}$ 则为 $\mathrm{Fe}_{3} \mathrm{O}_{4}$ 的 $2 \mathrm{p}_{3 / 2}{ }^{[18,25]}$. 由表 1 可以看出, 

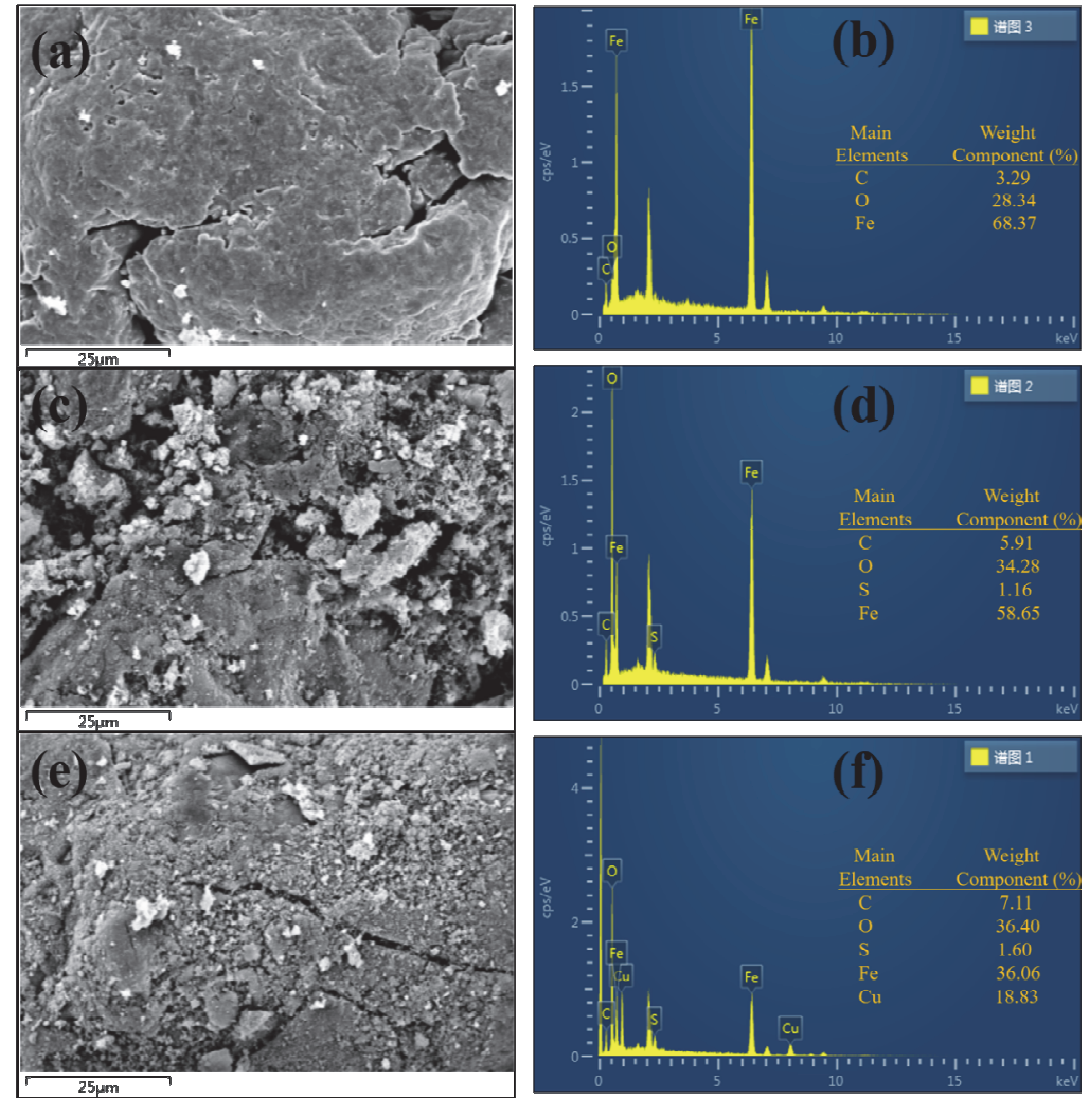

图 1 ZVI 的 SEM 图像和 EDS 谱图: (a), (b)反应前的 ZVI; (c), (d)在 $\mathrm{ZVI} / \mathrm{H}_{2} \mathrm{O}_{2}$ 体系中反应后; (e), (f) 在 $\mathrm{ZVI} / \mathrm{H}_{2} \mathrm{O}_{2}-\mathrm{Cu}$ 体系中反应后

Figure 1 SEM images and EDS spectra of ZVI: (a), (b) pristine ZVI; (c), (d) after reaction in $\mathrm{ZVI} / \mathrm{H}_{2} \mathrm{O}_{2}$ system; (e), (f) after reaction in $\mathrm{ZVI} / \mathrm{H}_{2} \mathrm{O}_{2}-\mathrm{Cu}$ system

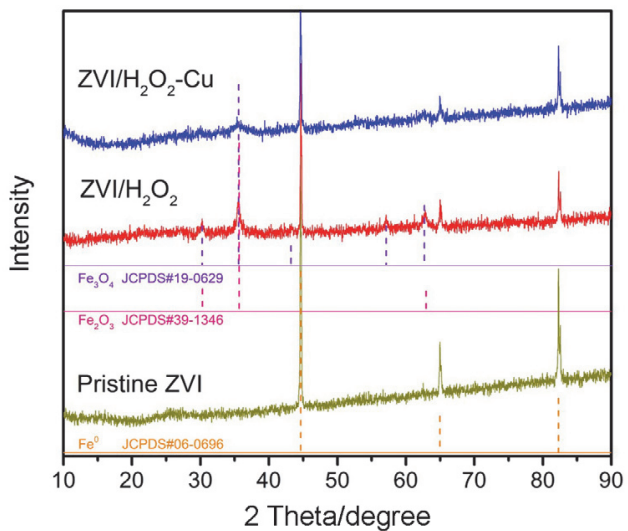

图 $2 \mathrm{ZVI}$ 反应前后的 XRD 谱图

Figure 2 XRD patterns of ZVI before and after reaction

$\mathrm{ZVI} / \mathrm{H}_{2} \mathrm{O}_{2}$ 体系中的 $\mathrm{Fe} 2 \mathrm{p}$ 峰面积均比 $\mathrm{ZVI} / \mathrm{H}_{2} \mathrm{O}_{2}-\mathrm{Cu}$ 体系 大, 再次证明了 $\mathrm{ZVI} / \mathrm{H}_{2} \mathrm{O}_{2}$ 体系中 ZVI 表面生成的氧化 产物比 ZVI/ $\mathrm{H}_{2} \mathrm{O}_{2}-\mathrm{Cu}$ 体系多. 同时, 相比于 $\mathrm{ZVI} / \mathrm{H}_{2} \mathrm{O}_{2}$ 体 系, $\mathrm{ZVI} / \mathrm{H}_{2} \mathrm{O}_{2}-\mathrm{Cu}$ 体系中 $716.7 \mathrm{eV}$ 处的 $\mathrm{Fe}_{2} \mathrm{O}_{3}$ 的卫星峰消 失了, 且 $\mathrm{Fe}_{3} \mathrm{O}_{4}$ 的峰面积占比增加, 表明 $\mathrm{Cu}^{2+}$ 加入 $\mathrm{ZVI} / \mathrm{H}_{2} \mathrm{O}_{2}$ 体系会使 ZVI 表面腐蚀产物 $\mathrm{Fe}_{3} \mathrm{O}_{4}$ 增加, $\mathrm{Fe}_{2} \mathrm{O}_{3}$ 相对减少.
图 3(c)为 $\mathrm{Cu} 2 \mathrm{p}$ 的 XPS 谱图, 如图所示, 在 $952.7 \mathrm{eV}$ 和 $942.0 \mathrm{eV}$ 的特征峰分别是 $\mathrm{CuO}$ 的 $2 \mathrm{p}_{1 / 2}$ 和 $2 \mathrm{p}_{3 / 2}$, 而 $932.7 \mathrm{eV}$ 处的特征峰则是 $\mathrm{Cu}^{0}$ 的 $2 p_{3 / 2}{ }^{\text {[26] }}$. 同时可以看出 $\mathrm{Cu}^{0}$ 的 $2 \mathrm{p}_{3 / 2}$ 峰面积远远大于其他两个峰. 由此可以分析 得出, $\mathrm{Cu}^{2+}$ 的还原产物主要是 $\mathrm{Cu}^{0}$, 同时还伴随有部分 $\mathrm{CuO}$ 产生.

\subsection{FTIR 表征}

图 4 为不同 ZVI 样品在 $400 \sim 4000 \mathrm{~cm}^{-1}$ 波段的红 外光谱. 相比于反应前的 ZVI, $\mathrm{ZVI} / \mathrm{H}_{2} \mathrm{O}_{2}$ 体系的 ZVI 在 红外波段 $472 \mathrm{~cm}^{-1}$ 和 $595 \mathrm{~cm}^{-1}$ 处均有较大的吸收, 但是 $\mathrm{ZVI} / \mathrm{H}_{2} \mathrm{O}_{2}-\mathrm{Cu}$ 体系在 $472 \mathrm{~cm}^{-1}$ 有较大的吸收而在 595 $\mathrm{cm}^{-1}$ 吸收较小. 研究表明, 红外波段 $472 \mathrm{~cm}^{-1}$ 和 595 $\mathrm{cm}^{-1}$ 与 $\mathrm{Fe}-\mathrm{O}$ 对应，且根据 $\mathrm{Li}^{[21]}$ 和 $\mathrm{Luo}^{[27]}$ 等的研究分 析，两处红外波段吸收分别为 $\mathrm{Fe}_{3} \mathrm{O}_{4}$ 和 $\mathrm{Fe}_{2} \mathrm{O}_{3}$ 中的 $\mathrm{Fe}-\mathrm{O}$ 振动 ${ }^{[28]}$. 该现象也证明了 $\mathrm{Cu}^{2+}$ 加入 $\mathrm{ZVI} / \mathrm{H}_{2} \mathrm{O}_{2}$ 体系会导 致 ZVI 表面腐蚀产物 $\mathrm{Fe}_{2} \mathrm{O}_{3}$ 减少, 符合 XPS 分析结果. 此外, 3427 和 $1641 \mathrm{~cm}^{-1}$ 两处对应 $\mathrm{O}-\mathrm{H}$, 可能是 ZVI 表 面吸附的空气中少量的水, $1031 \mathrm{~cm}^{-1}$ 处的吸收是 $\mathrm{C}$ $\mathrm{O}^{[23]}$. 此外 $\mathrm{ZVI} / \mathrm{H}_{2} \mathrm{O}_{2}-\mathrm{Cu}$ 体系反应后的 ZVI 在 1107 $\mathrm{cm}^{-1}$ 处多了一个吸收峰, 根据 Gotić 等 ${ }^{[29]}$ 研究报道可能 是一种新的铁氧化物 $\mathrm{Fe}_{8} \mathrm{O}_{8}(\mathrm{OH})_{6} \mathrm{SO}_{4}$. 

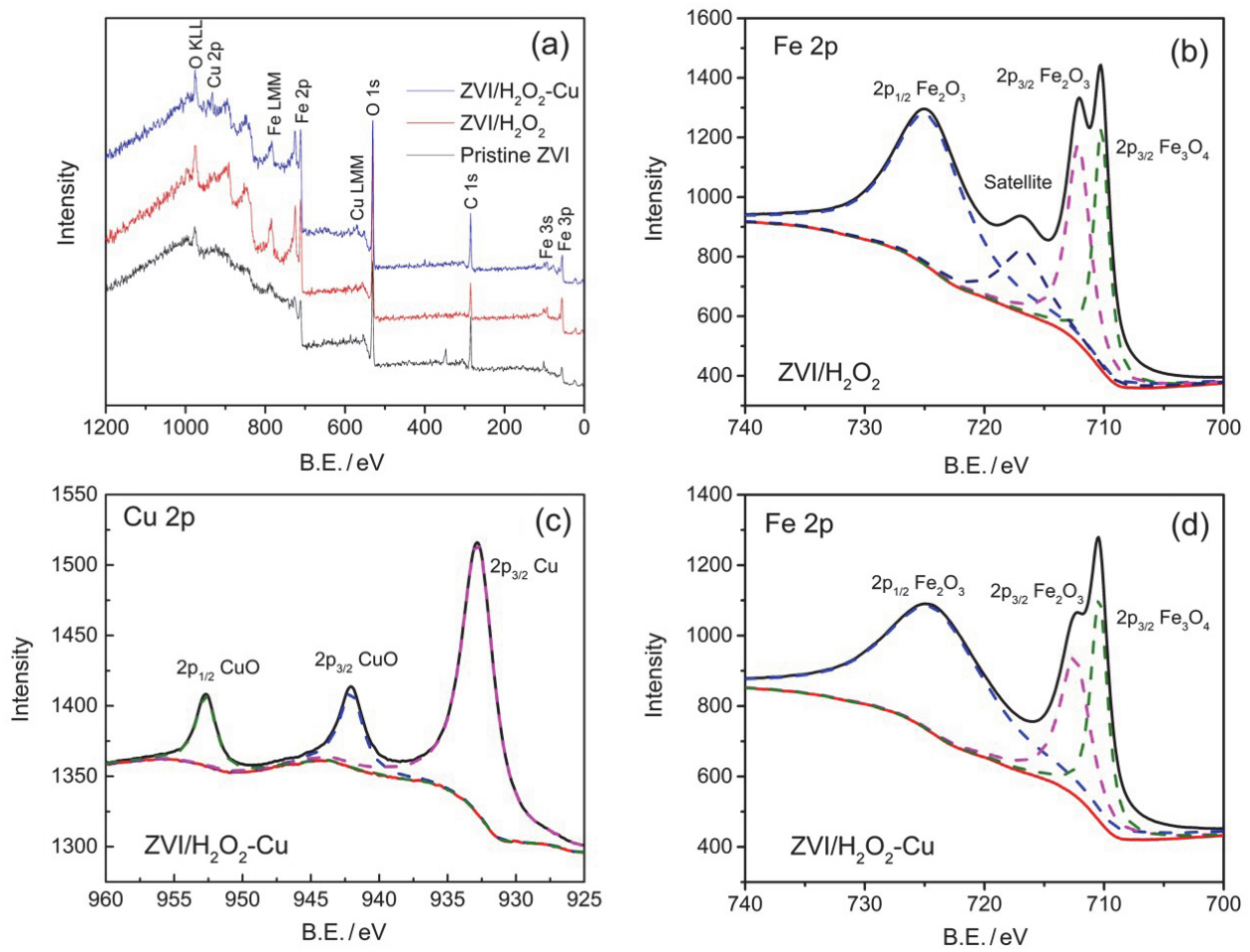

图 3 ZVI 反应前后的 XPS 谱图: (a) 全谱图; (b) ZVI/ $\mathrm{H}_{2} \mathrm{O}_{2}$ 体系的 $\mathrm{Fe} 2 \mathrm{p}$ 谱图; $\mathrm{ZVI} / \mathrm{H}_{2} \mathrm{O}_{2}-\mathrm{Cu}$ 体系的(c) $\mathrm{Cu} 2 \mathrm{p}$ 和(d) $\mathrm{Fe} 2 \mathrm{p}$ 谱图

Figure 3 XPS spectra of ZVI before and after reaction: (a) full-scan spectra; (b) $\mathrm{Fe} 2 \mathrm{p}$ in the $\mathrm{ZVI} / \mathrm{H}_{2} \mathrm{O}_{2}$ system; (c) $\mathrm{Cu} 2 \mathrm{p}$ and (d) Fe 2p in the $\mathrm{ZVI} / \mathrm{H}_{2} \mathrm{O}_{2}-\mathrm{Cu}$ system

表 $1 \mathrm{Fe}, \mathrm{Cu}$ 元素的化学状态及参数

Table 1 Chemical states of $\mathrm{Fe}, \mathrm{Cu}$ elements and parameters

\begin{tabular}{|c|c|c|c|c|c|}
\hline \multirow{2}{*}{$\begin{array}{l}\text { Element (photo } \\
\text { electro core level) }\end{array}$} & \multicolumn{2}{|c|}{ Peak position/eV } & \multirow{2}{*}{ Assignment } & \multirow{2}{*}{ Area } & \multirow{2}{*}{$\mathrm{FWHM} / \mathrm{eV}$} \\
\hline & $\mathrm{ZVI} / \mathrm{H}_{2} \mathrm{O}_{2}$ & $\mathrm{ZVI} / \mathrm{H}_{2} \mathrm{O}_{2}-\mathrm{Cu}$ & & & \\
\hline \multirow[t]{7}{*}{$\mathrm{Fe} 2 \mathrm{p}$} & 724.1 & & $2 \mathrm{p}_{1 / 2} \mathrm{Fe}_{2} \mathrm{O}_{3}$ & 5473.1 & 7.323 \\
\hline & 716.7 & & Satellite of $\mathrm{Fe}_{2} \mathrm{O}_{3}$ & 1373.7 & 4.47 \\
\hline & 712.1 & & $2 \mathrm{p}_{3 / 2} \mathrm{Fe}_{2} \mathrm{O}_{3}$ & 2281.4 & 2.333 \\
\hline & 710.2 & & $2 \mathrm{p}_{3 / 2} \mathrm{Fe}_{3} \mathrm{O}_{4}$ & 1825.9 & 1.539 \\
\hline & & 724.1 & $2 \mathrm{p}_{1 / 2} \mathrm{Fe}_{2} \mathrm{O}_{3}$ & 5264.5 & 9.995 \\
\hline & & 712.1 & $2 \mathrm{p}_{3 / 2} \mathrm{Fe}_{2} \mathrm{O}_{3}$ & 1735.5 & 3013 \\
\hline & & 710.2 & $2 \mathrm{p}_{3 / 2} \mathrm{Fe}_{3} \mathrm{O}_{4}$ & 1584.5 & 1.707 \\
\hline \multirow[t]{3}{*}{$\mathrm{Cu} 2 \mathrm{p}$} & - & 952.7 & $2 \mathrm{p}_{1 / 2} \mathrm{CuO}$ & 128.8 & 1.745 \\
\hline & - & 942.0 & $2 \mathrm{p}_{3 / 2} \mathrm{CuO}$ & 160.1 & 2.025 \\
\hline & - & 932.7 & $2 \mathrm{p}_{3 / 2} \mathrm{Cu}^{0}$ & 794.2 & 2.808 \\
\hline
\end{tabular}

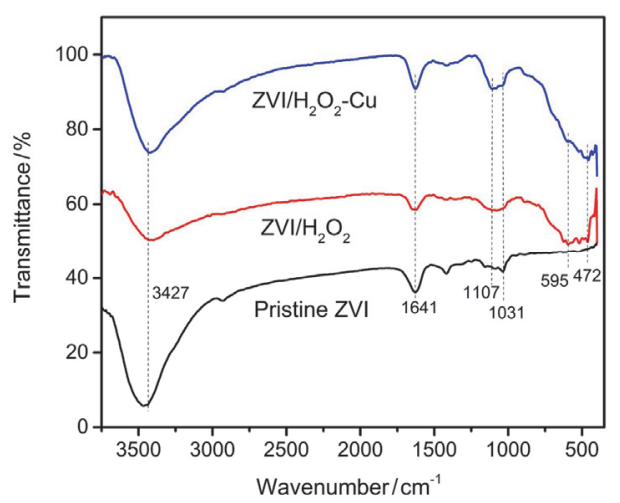

图 4 ZVI 反应前后的 FTIR 谱图

Figure 4 FTIR spectra of ZVI before and after reaction
根据 SEM, EDS, XRD, XPS 和 FTIR 的表征结果，表 明反应前的 ZVI 表面光滑仅有一层薄薄的氧化层. 经过 $\mathrm{ZVI} / \mathrm{H}_{2} \mathrm{O}_{2}$ 体系反应后的 ZVI发生明显地腐蚀, 且腐蚀产 物主要是 $\mathrm{Fe}_{3} \mathrm{O}_{4}$ 和 $\mathrm{Fe}_{2} \mathrm{O}_{3}$ 两种物质. 而在 $\mathrm{ZVI} / \mathrm{H}_{2} \mathrm{O}_{2}-\mathrm{Cu}$ 体系中，ZVI 表面腐蚀作用更加显著，但残留在 ZVI 表 面的腐蚀产物总量较少, 且腐蚀产物中 $\mathrm{Fe}_{2} \mathrm{O}_{3}$ 占比相对 减少, $\mathrm{Fe}_{3} \mathrm{O}_{4}$ 占比增加. 主要原因是在反应过程中, ZVI 与 $\mathrm{Cu}^{2+}$ 发生的置换作用能促进 ZVI表面腐蚀, 并将置换 的 $\mathrm{Fe}^{2+}$ 直接释放在反应液中, 进而通过 Fenton 反应生成 • OH，从而削弱了直接在 ZVI 表面发生酸腐蚀作用. 此 外, $\mathrm{Cu}^{2+}$ 得到电子还原成的 $\mathrm{Cu}^{0}$ 以及与 $\mathrm{H}_{2} \mathrm{O}_{2}$ 反应生成的 $\mathrm{CuO}$, 均在 ZVI 表面沉积. 


\section{$2.5 \mathrm{pH}$ 的影响}

$\mathrm{pH}$ 是对 Fenton 以及类 Fenton 体系去除目标物影响 很大的因素, 对比研究了 $\mathrm{ZVI}, \mathrm{ZVI}-\mathrm{Cu}, \mathrm{H}_{2} \mathrm{O}_{2}-\mathrm{Cu}$, $\mathrm{ZVI} / \mathrm{H}_{2} \mathrm{O}_{2}$ 和 $\mathrm{ZVI} / \mathrm{H}_{2} \mathrm{O}_{2}-\mathrm{Cu}$ 五个体系在 $\mathrm{pH}$ 为 $2.5 \sim 8.5$ 的 范围内对 $\mathrm{MB}$ 和 $\mathrm{TCu}$ 的去除, 以及总铁离子 $(\mathrm{TFe})$ 溶出 情况的影响. 图 5(a)和(c)分别为五个体系在不同 $\mathrm{pH}$ 条 件下对 MB 的降解图和 TFe 溶出浓度图. 如图所示, 单 独的 ZVI 体系在 $\mathrm{pH}$ 为 2.5 的条件下, 对 $\mathrm{MB}$ 的降解达 到 $80 \%$. 因为在强酸条件下, ZVI 会与溶解氧反应原位 生成 $\mathrm{H}_{2} \mathrm{O}_{2}$ (方程(1)), 同时释放大量 $\mathrm{Fe}^{2+}$ (图 5(d)), 通过 Fenton 反应产生 $\cdot \mathrm{OH}$ 实现 $\mathrm{MB}$ 的降解. 然而单独的 ZVI-Cu 体系即使在较低 $\mathrm{pH}$ 下对 $\mathrm{MB}$ 的降解作用也比较 小, 因为大量的 $\mathrm{ZVI}$ 与 $\mathrm{Cu}^{2+}$ 直接发生了置换反应, 导致 原位生成氧化剂 $\mathrm{H}_{2} \mathrm{O}_{2}$ 作用减小. 而 $\mathrm{H}_{2} \mathrm{O}_{2}-\mathrm{Cu}$ 体系对于 $\mathrm{MB}$ 的降解在强酸性条件下有微弱作用, 主要由于在强 酸条件下 $\mathrm{H}_{2} \mathrm{O}_{2}$ 的氧化性增强; 然而随着 $\mathrm{pH}$ 上升, 对 $\mathrm{MB}$ 几乎没有降解作用. 相比于前三个体系, 随着 $\mathrm{H}_{2} \mathrm{O}_{2}$ 的加入 $\mathrm{ZVI} / \mathrm{H}_{2} \mathrm{O}_{2}$ 体系在 $\mathrm{pH}$ 为 $2.5 \sim 4.5$ 的范围内都展现 较强的去除 $\mathrm{MB}$ 的作用. 由图 5(a)可以看出, 对于 $\mathrm{ZVI} / \mathrm{H}_{2} \mathrm{O}_{2}-\mathrm{Cu}$ 体系, $\mathrm{Cu}^{2+}$ 的加入不仅强化了 $\mathrm{ZVI} / / \mathrm{H}_{2} \mathrm{O}_{2}$ 体 系对 $\mathrm{MB}$ 的降解, 同时还将该体系有效 $\mathrm{pH}$ 范围扩大到 了 5.5. 其主要原因是 $\mathrm{Cu}^{2+}$ 与 ZVI 的置换作用, 能减少
ZVI 表面氧化产物的生成, 促进 $\mathrm{Fe}^{2+}$ 的释放, 强化芬顿 反应.

图 5(b)展现了不同 $\mathrm{pH}$ 对 $\mathrm{ZVI} / \mathrm{Cu}$ 与 $\mathrm{ZVI} / \mathrm{H}_{2} \mathrm{O}_{2}-\mathrm{Cu}$ 体系去除 $\mathrm{TCu}$ 的影响. 随着 $\mathrm{pH}$ 提高, 两个体系对 $\mathrm{TCu}$ 的去除呈现相同的趋势, 先增加后减小. 在 $\mathrm{pH}=2.5$ 时, $\mathrm{TCu}$ 的去除都比较低, 一方面是因为在 $\mathrm{pH}=2.5$ 的条件 下, 由图 5(c) 可知, 大部分的 ZVI 被强酸腐蚀( TFe 约为 $1.2 \mathrm{mmol} / \mathrm{L}=67.2 \mathrm{mg} / \mathrm{L}, \mathrm{ZVI}$ 投量为 $100 \mathrm{mg} / \mathrm{L}$, 腐蚀比 例高达 $67.2 \%$ )(方程(2)), 导致可用于 $\mathrm{Cu}^{2+}$ 还原的 ZVI 的量大大减少; 另一方面, 研究表明在 $\mathrm{pH}$ 为 2.5 的条件 下, 还原产生的 $\mathrm{Cu}^{0}$ 也会与 $\mathrm{H}^{+}$重新反应溶解(方程(3)). 但是, 当 $\mathrm{pH}$ 大于 3.5 之后, $\mathrm{TCu}$ 的去除开始逐渐降低. 主要是因为随着 $\mathrm{pH}$ 升高, ZVI 表面氧化层腐蚀较慢, 此 外溶解出来的 $\mathrm{Fe}^{2+}$ 和 $\mathrm{Fe}^{3+}$ 在中性和碱性条件下还会迅 速生成氢氧化物附着在 ZVI 表面 ${ }^{[30]}$, 阻碍了 ZVI 与 $\mathrm{Cu}^{2+}$ 的接触, 降低了 $\mathrm{TCu}$ 的去除. 相比于 $\mathrm{ZVI} / \mathrm{Cu}$ 体系, $\mathrm{ZVI} / \mathrm{H}_{2} \mathrm{O}_{2}-\mathrm{Cu}$ 体系对 $\mathrm{TCu}$ 的去除率要低一点, 是由于 $\mathrm{H}_{2} \mathrm{O}_{2}$ 的氧化作用会将还原产物 $\mathrm{Cu}^{0}$ 再次氧化为 $\mathrm{Cu}^{+}$和 $\mathrm{Cu}^{2+}$.

$$
\begin{aligned}
& \mathrm{Fe}^{0}+\mathrm{O}_{2}+2 \mathrm{H}^{+} \rightarrow \mathrm{Fe}^{2+}+\mathrm{H}_{2} \mathrm{O}_{2} \\
& \mathrm{Fe}^{0}+2 \mathrm{H}^{+} \rightarrow \mathrm{Fe}^{2+}+\mathrm{H}_{2} \\
& \mathrm{Cu}^{0}+2 \mathrm{H}^{+} \rightarrow \mathrm{Cu}^{2+}+\mathrm{H}_{2}
\end{aligned}
$$
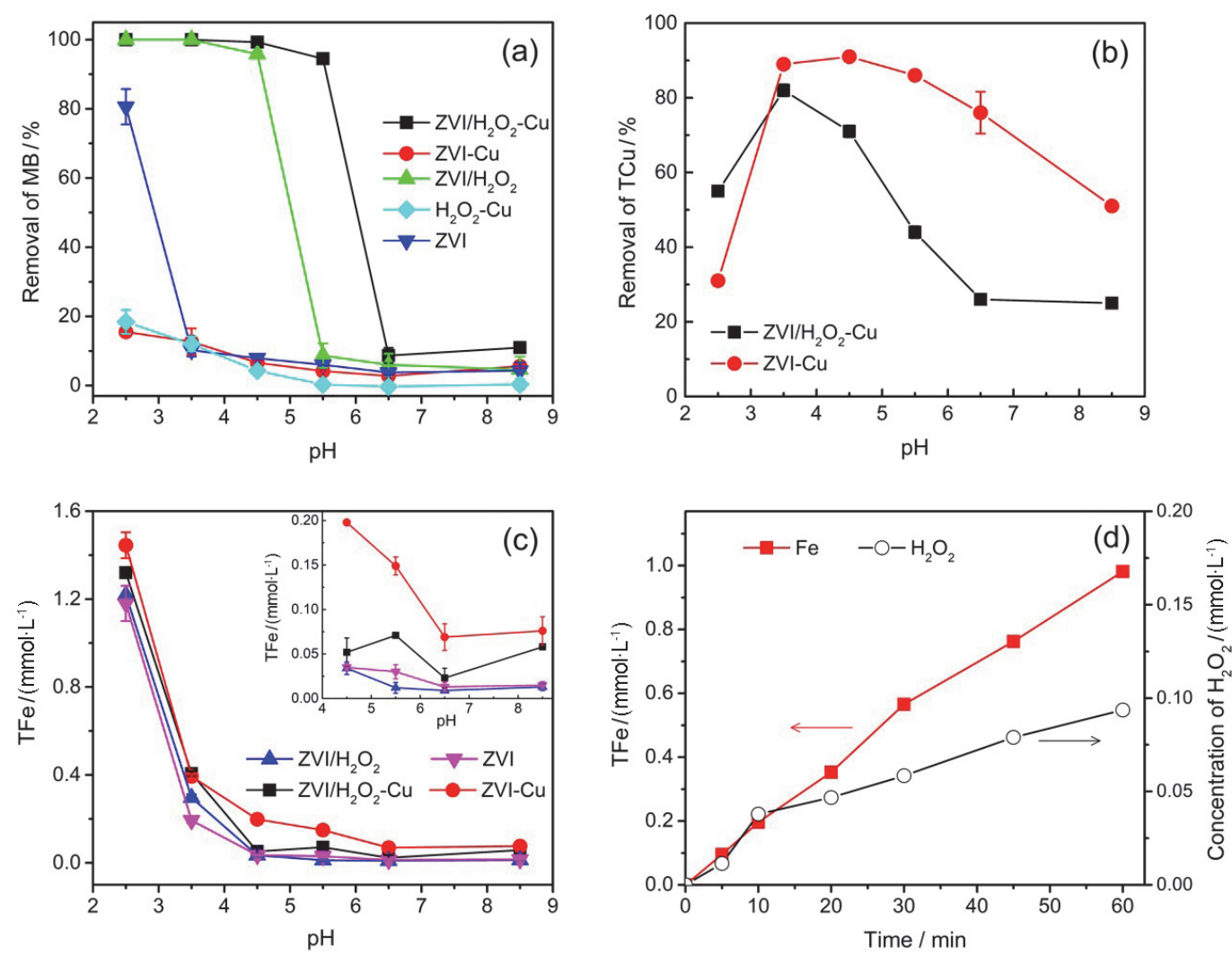

图 $5 \mathrm{pH}$ 对 $\mathrm{ZVI} / \mathrm{H}_{2} \mathrm{O}_{2}-\mathrm{Cu}, \mathrm{ZVI} / \mathrm{H}_{2} \mathrm{O}_{2}, \mathrm{ZVI}-\mathrm{Cu}, \mathrm{H}_{2} \mathrm{O}_{2}-\mathrm{Cu}$ 和 $\mathrm{ZVI}$ 体系的影响: (a) 去除 $\mathrm{MB}$; (b) 去除 $\mathrm{TCu}$; (c) $\mathrm{TFe}$ 的浓度; (d) $\mathrm{pH}=2.5$ 时 $\mathrm{ZVI}$ 体系中 $\mathrm{TFe}$ 和 $\mathrm{H}_{2} \mathrm{O}_{2}$ 的浓度变化

Figure 5 The effect of initial $\mathrm{pH}$ on the $\mathrm{ZVI} / \mathrm{H}_{2} \mathrm{O}_{2}-\mathrm{Cu}, \mathrm{ZVI} / \mathrm{H}_{2} \mathrm{O}_{2}$, ZVI-Cu, $\mathrm{H}_{2} \mathrm{O}_{2}-\mathrm{Cu}$ and ZVI system. (a) removal of MB; (b) removal of TCu; (c) concentration of TFe; (d) concentration changes of $\mathrm{TFe}$ and $\mathrm{H}_{2} \mathrm{O}_{2}$ in the $\mathrm{ZVI}$ system at $\mathrm{pH}=2.5$ 


\section{6 自由基捕获}

芬顿体系和类芬顿体系中的主要活性氧物种为

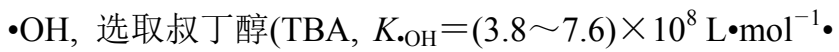
$\left.\mathrm{s}^{-1}\right)$ 作为 $\cdot \mathrm{OH}$ 捕获剂 ${ }^{[19]}$, 图 6 为在不同 $\mathrm{TBA}$ 浓度条件下 的 $\mathrm{MB}$ 的降解图. 当 $\mathrm{TBA}$ 浓度由 0 增加到 $10 \mathrm{mmol} / \mathrm{L}$ 时, MB 的降解受到了明显的抑制, 然而当 TBA 浓度大 于 $10 \mathrm{mmol} / \mathrm{L}$ 之后, 对于 $\mathrm{MB}$ 降解抑制作用增加很小, 表明体系中产生了 $\cdot \mathrm{OH}$, 且 $10 \mathrm{mmol} / \mathrm{L}$ 的捕获剂就能将 体系中・OH 完全捕获.

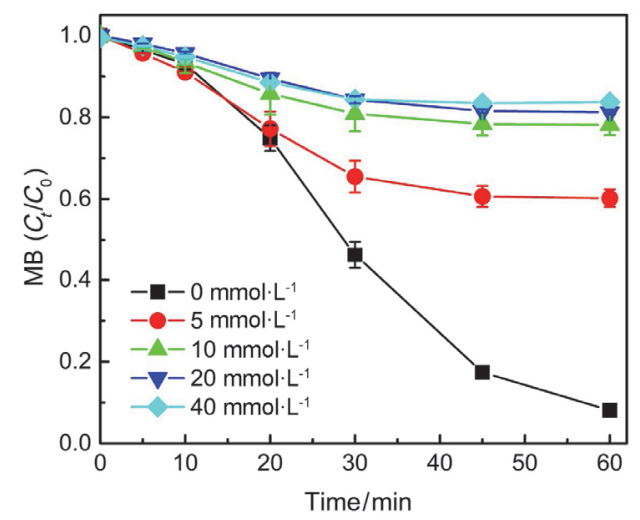

图 6 叔丁醇浓度对 $\mathrm{MB}$ 降解的影响

Figure 6 The effect of TBA concentration on the removal of MB

\section{7 机理分析}

图 7 为 $\mathrm{ZVI} / \mathrm{H}_{2} \mathrm{O}_{2}$ 体系协同去除 $\mathrm{TCu}$, 强化 $\mathrm{MB}$ 降解 机理图. $\mathrm{Cu}^{2+}$ 与 $\mathrm{ZVI} / \mathrm{H}_{2} \mathrm{O}_{2}$ 的协同机理主要分为两方面: 一方面, $\mathrm{Cu}^{2+}$ 加速 ZVI 表面 $\mathrm{Fe}^{2+}$ 溶出, 强化 Fenton 和 Fenton-like 反应, 加速生成 $\cdot \mathrm{OH}$ 氧化 $\mathrm{MB}$; 另一方面, $\mathrm{Fe}$ 和 $\mathrm{Cu}$ 的氧化还原循环反应能持续生成活化 $\mathrm{H}_{2} \mathrm{O}_{2}$ 的 $\mathrm{Fe}^{2+}$ 和 $\mathrm{Cu}^{+}$. 详细过程如下.

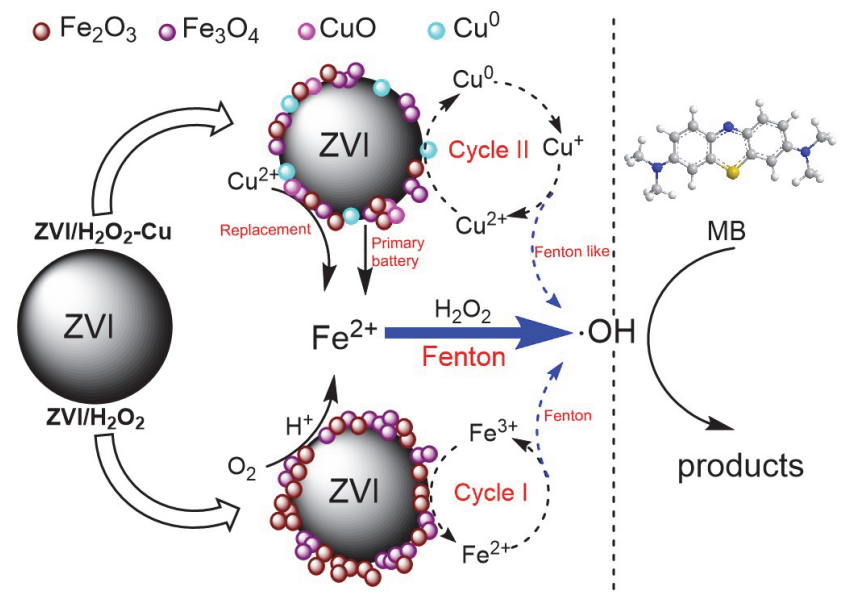

图 $7 \mathrm{ZVI} / \mathrm{H}_{2} \mathrm{O}_{2}-\mathrm{Cu}$ 体系协同去除 $\mathrm{TCu}$ 和 $\mathrm{MB}$ 的机理图

Figure 7 The mechanism for synergistic removal of $\mathrm{TCu}$ and $\mathrm{MB}$ by the $\mathrm{ZVI} / \mathrm{H}_{2} \mathrm{O}_{2}-\mathrm{Cu}$ system

ZVI-Fenton 反应: (1) ZVI 可与溶解氧在酸性条件下 反应缓慢释放 $\mathrm{Fe}^{2+}$, 生成的 $\mathrm{Fe}^{2+}$ 催化 $\mathrm{H}_{2} \mathrm{O}_{2}$ 产生 $\cdot \mathrm{OH}$ (方
程(1), (2), (4)). (2) $\mathrm{Cu}^{2+}$ 加入可与 ZVI 发生置换反应(方 程(5)), 加速 $\mathrm{Fe}^{2+}$ 的释放, 强化 Fenton 反应. (3) 还原产 物 $\mathrm{Cu}^{0}$ 附着在 ZVI 表面, 形成微小原电池, 促进电子快 速转移，从而持续腐蚀 ZVI 表面释放 $\mathrm{Fe}^{2+}$.

$\mathrm{Cu}^{+}$-Fenton-like 反应: $\mathrm{Cu}^{+}$也可催化 $\mathrm{H}_{2} \mathrm{O}_{2}$ 产生・ $\mathrm{OH}$ (方程(6)).

两个氧化还原循环: (1) ZVI 释放 $\mathrm{Fe}^{2+}, \mathrm{Fe}^{2+}$ 被氧化 为 $\mathrm{Fe}^{3+}$ (方程(4), (7)), $\mathrm{Fe}^{3+}$ 可再次被 $\mathrm{ZVI}$ 还原为 $\mathrm{Fe}^{2+}$ (方 程(8)), 从而形成 $\mathrm{Fe}$ 的氧化还原循环. (2) $\mathrm{Cu}^{2+}$ 被 ZVI 还 原为 $\mathrm{Cu}^{0}, \mathrm{Cu}^{0}$ 与 $\mathrm{H}_{2} \mathrm{O}_{2}$ 反应被氧化为 $\mathrm{Cu}^{+}$(方程(9)), $\mathrm{Cu}^{+}$ 再通过类芬顿反应被氧化为 $\mathrm{Cu}^{2+}$ ，从而形成 $\mathrm{Cu}$ 氧化还 原循环.

$\mathrm{Fe}, \mathrm{Cu}$ 化学转化: (1) 关于 $\mathrm{Fe}$ 的化学转化, 一方面, $\mathrm{Fe}^{0}$ 可直接与 $\mathrm{O}_{2}$ 反应生成 $\mathrm{Fe}_{3} \mathrm{O}_{4}$ 和 $\mathrm{Fe}_{2} \mathrm{O}_{3}$ (方程(10) $)^{[31]}$. 另一方面, 溶液中的 $\mathrm{Fe}^{3+}$ 生成的 $\mathrm{Fe}(\mathrm{OH})_{3}$ 也可和 $\mathrm{Fe}^{2+}$ 反 应生成 $\mathrm{Fe}_{3} \mathrm{O}_{4}$ (方程(11), (12)). 此外, $\mathrm{Fe}_{2} \mathrm{O}_{3}$ 也能向 $\mathrm{Fe}_{3} \mathrm{O}_{4}$ 发生转化(方程(13), (14) $)^{[32]}$. (2) 关于 $\mathrm{Cu}$ 的化学转化, 溶液中的 $\mathrm{Cu}^{2+}$ 可与水中 $\mathrm{H}_{2} \mathrm{O}_{2}$ 反应生成 $\mathrm{CuO}$ (方程(15), $(16))^{[33]}$, 同时还原产生的 $\mathrm{Cu}^{0}$ 可直接与 $\mathrm{O}_{2}$ 反应生成 $\mathrm{CuO}($ 方程(17)).

$$
\begin{aligned}
& \mathrm{Fe}^{2+}+\mathrm{H}_{2} \mathrm{O}_{2} \rightarrow \mathrm{Fe}^{3+}+\cdot \mathrm{OH}+\mathrm{OH}^{-} \\
& \mathrm{Fe}^{0}+\mathrm{Cu}^{2+} \rightarrow \mathrm{Cu}^{0}+\mathrm{Fe}^{2+} \\
& \mathrm{Cu}^{+}+\mathrm{H}_{2} \mathrm{O}_{2} \rightarrow \mathrm{Cu}^{2+}+\cdot \mathrm{OH}+\mathrm{OH}^{-} \\
& \mathrm{Fe}^{2+}+\mathrm{O}_{2} \rightarrow \mathrm{Fe}^{3+}+\mathrm{O}_{2}^{--} \\
& 2 \mathrm{Fe}^{3+}+\mathrm{Fe}^{0} \rightarrow 3 \mathrm{Fe}^{2+} \\
& 2 \mathrm{Cu}^{0}+\mathrm{H}_{2} \mathrm{O}_{2} \rightarrow 2 \mathrm{Cu}^{+}+2 \mathrm{OH}^{-} \\
& 5 \mathrm{Fe}^{0}+\mathrm{H}_{2} \mathrm{O}+3 \mathrm{O}_{2} \rightarrow \mathrm{Fe}_{2} \mathrm{O}_{3}+\mathrm{Fe}_{3} \mathrm{O}_{4}+\mathrm{H}_{2} \\
& \mathrm{Fe}^{3+}+3 \mathrm{H}_{2} \mathrm{O} \rightarrow \mathrm{Fe}(\mathrm{OH})_{3}+3 \mathrm{H}^{+} \\
& 2 \mathrm{Fe}(\mathrm{OH})_{3}+\mathrm{Fe}^{2+} \rightarrow \mathrm{Fe}_{3} \mathrm{O}_{4}+2 \mathrm{H}_{2} \mathrm{O}+2 \mathrm{H}^{+} \\
& \mathrm{Fe}_{2} \mathrm{O}_{3}+\mathrm{H}_{2} \mathrm{O} \rightarrow 2 \mathrm{FeOOH} \\
& 2 \mathrm{FeOOH}^{+} \mathrm{Fe}^{2+} \rightarrow \mathrm{Fe}_{3} \mathrm{O}_{4}+2 \mathrm{H}^{+} \\
& \mathrm{Cu}^{2+}+\mathrm{H}_{2} \mathrm{O}_{2} \rightarrow \mathrm{CuOOH}{ }^{+}+\mathrm{H}^{+} \\
& 2 \mathrm{CuOOH}^{+} \rightarrow 2 \mathrm{CuO}+\mathrm{O}_{2}+2 \mathrm{H}^{+} \\
& 2 \mathrm{Cu}^{0}+\mathrm{O}_{2} \rightarrow 2 \mathrm{CuO}
\end{aligned}
$$

\section{3 结论}

$\mathrm{ZVI} / \mathrm{H}_{2} \mathrm{O}_{2}-\mathrm{Cu}$ 体系可以同时有效地去除 $\mathrm{TCu}$ 和 $\mathrm{MB}$. $\mathrm{ZVI} / \mathrm{H}_{2} \mathrm{O}_{2}$ 体系腐蚀产物主要是 $\mathrm{Fe}_{3} \mathrm{O}_{4}$ 和 $\mathrm{Fe}_{2} \mathrm{O}_{3}$, 且腐蚀 产物总量较多. 而相比于 $\mathrm{ZVI} / \mathrm{H}_{2} \mathrm{O}_{2}$ 体系, $\mathrm{ZVI} / \mathrm{H}_{2} \mathrm{O}_{2}-\mathrm{Cu}$ 体系腐蚀产物总量减少, 且 $\mathrm{Cu}^{2+}$ 的加入会导致体系中 $\mathrm{Fe}_{3} \mathrm{O}_{4}$ 占比相对增加. $\mathrm{TCu}$ 去除过程中生成的 $\mathrm{Cu}^{0}$ 和 $\mathrm{CuO}$ 附着在 ZVI 表面. $\mathrm{ZVI} / \mathrm{H}_{2} \mathrm{O}_{2}-\mathrm{Cu}$ 体系主要通过 ZVI 与 $\mathrm{Cu}^{2+}$ 置换作用, $\mathrm{ZVI}$ 和 $\mathrm{Cu}^{0}$ 的原电池效应以及酸性腐蚀 作用来释放 $\mathrm{Fe}^{2+}$, 进而通过 $\mathrm{Fe}^{2+}$-Fenton 和 $\mathrm{Cu}^{+}$-Fentonlike 产生・OH 降解 $\mathrm{MB}$. 此外, $\mathrm{ZVI} / \mathrm{H}_{2} \mathrm{O}_{2}-\mathrm{Cu}$ 体系相比于 $\mathrm{ZVI} / \mathrm{H}_{2} \mathrm{O}_{2}$ 体系在降解 $\mathrm{MB}$ 时, 有效 $\mathrm{pH}$ 可以扩大到 5.5. 
ZVI-Fenton 体系可有效地还原去除水环境中重金属离 子, 同时强化对水体中有机污染物的氧化降解, 实现复 合污染物的协同去除，对处理含重金属和有机污染物的 工业废水有很好的理论指导作用.

\section{4 实验试剂和方法}

\section{1 实验试剂}

零价铁 $(\geqslant 99.9 \mathrm{wt} \%, 100$ 目)购自阿拉丁有限公司 (中国上海); 亚甲基蓝 $(\mathrm{MB}, \geqslant 98.5 \%$ ) 和硫酸铜 $\left(\mathrm{CuSO}_{4} \cdot 5 \mathrm{H}_{2} \mathrm{O}, \geqslant 99.0 \%\right)$ 购自西格玛奥德里奇有限公司 (中国上海); 叔丁醇(TBA)、过氧化氢 $\left(\mathrm{H}_{2} \mathrm{O}_{2}, 30 \%\right)$ 、硫酸 $\left(\mathrm{H}_{2} \mathrm{SO}_{4}\right)$ 、氢氧化钠 $(\mathrm{NaOH})$ 购自科龙化工有限公司(中国 成都), 所有试剂均为分析纯. 所有溶液均由去离子水 (DI)制备.

\section{2 实验仪器}

$\mathrm{pH}$ 计 (中国上海雷磁); 紫外-可见分光光度计 (MAPADA UV-1800); 总有机碳/总氮分析仪(TOC, multi N/C 3100 analyzer); 能谱仪(EDS, Octane super); 扫描电 镜(SEM, FEI-inspect F50); X 射线衍射(XRD, Bruker D8 Advance A25x); X 射线光电子能谱(XPS, XSAM 800); 傅里叶变换红外光谱仪(FT-IR, NXUS 670).

\section{3 实验方法}

所有实验均在玻璃烧杯中进行, 通过恒温水浴锅控 制反应温度为室温 $\left(25^{\circ} \mathrm{C}\right)$. 在每组反应中, 依次加入 $\mathrm{MB}(50 \mu \mathrm{mol} / \mathrm{L}) 、 \mathrm{Cu}^{2+}(0.2 \mathrm{mmol} / \mathrm{L})$ 和 $\mathrm{H}_{2} \mathrm{O}_{2}(1 \mathrm{mmol} / \mathrm{L})$ 试剂制备成体积为 $300 \mathrm{~mL}$ 的混合溶液, 然后用硫酸(1 $\mathrm{mol} / \mathrm{L})$ 和氢氧化钠 $(0.1 \mathrm{~mol} / \mathrm{L})$ 将溶液的 $\mathrm{pH}$ 值调整为所 需的值, 通过在机械搅拌器 $(250 \mathrm{r} / \mathrm{min})$ 下添加 ZVI (100 $\mathrm{mg} / \mathrm{L})$ 开始反应. 在每个时间间隔, 提取样品 $(5 \mathrm{~mL})$, 立 即通过 $0.22 \mu \mathrm{m}$ 纤维膜过滤, 并注入试管进行 $\mathrm{MB}$ 吸光 度, $\mathrm{TCu}$ 浓度和 $\mathrm{TFe}$ 浓度分析.

\section{$4.4 \mathrm{TCu}$ 和 MB 方法}

$\mathrm{TCu}$ 浓度检测方法: 参考 Zhou 等 ${ }^{[34]}$ 的方法, 做了 适当改进. 采用紫外 - 可见分光光度计 (Mapada $\mathrm{UV}-1800)$ 测定 $\mathrm{TCu}$ 的浓度. 首先, 配置 $50 \mathrm{mmol} / \mathrm{L}$ 抗坏 血酸和 $10 \mathrm{mmol} / \mathrm{L}$ 新亚铜试剂半水合物母液. 然后, 用 $5 \mathrm{~mL}$ 注射器提取反应液并通过 $0.22 \mu \mathrm{m}$ 纤维膜过滤. 将 过滤后的 $1 \mathrm{~mL}$ 样品加入含有 $0.4 \mathrm{~mL}$ 抗坏血酸、 $0.2 \mathrm{~mL}$ 新亚铜试剂半水合物母液和 $3.4 \mathrm{~mL}$ 去离子水混合, 平 衡 $60 \mathrm{~s}$ 后在波长 $\lambda=454 \mathrm{~nm}$ 处检测吸光度.

MB 浓度检测方法: 采用紫外-可见分光光度计 (Mapada UV-1800)测定 MB 的浓度. 用 $5 \mathrm{~mL}$ 注射器提取 反应液并通过 $0.22 \mu \mathrm{m}$ 纤维膜过滤. 然后将样品注入 10 $\mathrm{mm}$ 石英比色典, 在 $\mathrm{MB}$ 的最大吸收波长 $664 \mathrm{~nm}$ 处测定 吸光度.

\section{References}

[1] Fu, F.; Dionysiou, D. D.; Liu, H. J. Hazard. Mater. 2014, 267, 194.

[2] Yamaguchi, R.; Kurosu, S.; Suzuki, M.; Kawase, Y. Chem. Eng. J. 2018, 334, 1537.

[3] Wei, X. Y.; Gao, N. Y.; Li, C. J.; Deng, Y.; Zhou, S. Q.; Li, L. Chem. Eng. J. 2016, 285, 660 .

[4] Mirzaei, A.; Chen, Z.; Haghighat, F.; Yerushalmi, L. Chemosphere 2017, 174, 665 .

[5] Ling, R.; Chen, J. P.; Shao, J.; Reinhard, M. Water Res. 2018, 134, 44.

[6] Liu, J.; Ou, C.; Han, W.; Faheem, F.; Shen, J.; Bi, H.; Sun, X.; Li, J.; Wang, L. RSC Adv. 2015, 5, 57444.

[7] Cho, Y.; Choi, S. I. Chemosphere 2010, 81, 940.

[8] Zheng, Z. Q.; Lu, G. N.; Wang, R.; Huang, K. B.; Tao, X. Q.; Yang, Y. L.; Zou, M. Y.; Xie, Y. Y.; Yin, H. Environ. Pollut. 2019, 245, 780.

[9] Gu, T. H.; Shi, J. M.; Hua, Y. L.; Liu, J.; Wang, W.; Zhang, W. X. Acta Chim. Sinica 2017, 75, 991. (顾天航, 石君明, 滑熠龙, 刘静, 王伟, 张伟贤, 化学学报, 2017, 75, 991.)

[10] Li, Z.; Luo, S. Q.; Yang, Y.; Chen, J. W. Chemosphere 2019, 216, 499.

[11] Cai, C.; Wang, L. G.; Gao, H.; Hou, L. W.; Zhang, H. J. Environ. Sci. 2014, 26, 1267

[12] Cao, C. J.; Liu, X. G.; Ju, X. R.; Chen, X. R. Acta Phys.-Chim. Sin. 2013，29，2475. (曹崇江，刘晓庚，鞠兴荣，陈晓荣，物理化学学 报, 2013, 29, 2475.)

[13] Huang, X. Y.; Wang, W.; Lin, L.; Zhang, W. X. Acta Chim. Sinica 2017, 75, 529. (黄潇月, 王伟, 凌岗, 张伟贤, 化学学报, 2017, 75, 529.)

[14] Liu, J.; Gu, T. H.; Wang, W.; Liu, A. R.; Zhang, W. X. Acta Chim Sinica 2019, 77, 121. (刘静, 顾天航, 王伟, 刘爱荣, 张伟贤, 化 学学报, 2019, 77, 121.)

[15] Li, J. H.; Lin, C. F.; Qin, W.; Xiao, X. B.; Wei, L. Acta Phys.-Chim. Sin. 2016, 32, 2717. (李继红, 林常枫, 覃吴, 肖显斌, 魏利, 物理 化学学报, 2016, 32, 2717.)

[16] Jiang, X.; Qiao, J.; Lo, I. M.; Wang, L.; Guan, X.; Lu, Z.; Zhou, G.; Xu, C. J. Hazard. Mater. 2015, 283, 880.

[17] Fu, R. B.; Yang, Y. P.; Xu, Z.; Zhang, X.; Guo, X. P.; Bi, D. S. Chemosphere 2015, 138, 726 .

[18] Diao, Z.-H.; Xu, X.-R.; Jiang, D.; Liu, J.-J.; Kong, L.-J.; Li, G.; Zuo, L.-Z.; Wu, Q.-H. Chem. Eng. J. 2017, 315, 167.

[19] Liu, C. M.; Diao, Z. H.; Huo, W. Y.; Kong, L. J.; Du, J. J. Environ. Pollut. 2018, 239, 698.

[20] Sleiman, N.; Deluchat, V.; Wazne, M.; Mallet, M.; Courtin-Nomade, A.; Kazpard, V.; Baudu, M. Colloids Surf., A 2017, 514, 1.

[21] Li, H.; Wan, J.; Ma, Y.; Wang, Y.; Huang, M. Chem. Eng. J. 2014, $237,487$.

[22] Xu, C. H.; Zhang, B. L.; Wang, Y. H.; Shao, Q. Q.; Zhou, W. Z.; Fan, D. M.; Bandstra, J. Z.; Shi, Z. Q.; Tratnyek, P. G. Environ. Sci. Technol. 2016, 50, 11879 .

[23] Zhang, L.; Shao, Q.; Xu, C. J. Clean. Prod. 2019, 213, 753.

[24] Liu, W.; Sun, W.; Borthwick, A. G. L.; Wang, T.; Li, F.; Guan, Y. J. Hazard. Mater. 2016, 317, 385.

[25] Grosvenor, A. P.; Kobe, B. A.; Biesinger, M. C.; McIntyre, N. S Surf. Interface Anal. 2004, 36, 1564.

[26] Choi, K.; Lee, W. J. Hazard. Mater. 2012, 211-212, 146.

[27] Luo, F.; Chen, Z.; Megharaj, M.; Naidu, R. Chem. Eng. J. 2016, 294, 290.

[28] Gao, Y. Y.; Li, H. X.; Ou, Z. Z.; Hao, P.; Li, Y.; Yang, G. Q. Acta Phys.-Chim. Sin. 2011, 27, 2469. (高云燕, 李海霞, 欧植泽, 郝平, 李嫕, 杨国强, 物理化学学报, 2011, 27, 2469.)

[29] Gotic, M.; Music, S. J. Mol. Struct. 2007, 834, 445.

[30] Dong, H.; He, Q.; Zeng, G.; Tang, L.; Zhang, L.; Xie, Y.; Zeng, Y.; Zhao, F. Chem. Eng. J. 2017, 316, 410.

[31] Cai, X.; Gao, Y.; Sun, Q.; Chen, Z.; Megharaj, M.; Naidu, R. Chem. Eng. J. 2014, 244, 19.

[32] Guan, X.; Sun, Y.; Qin, H.; Li, J.; Lo, I. M.; He, D.; Dong, Water Res. 2015, 75, 224.

[33] Wang, N.; Zheng, T.; Jiang, J.; Wang, P. Chem. Eng. J. 2015, 260, 386.

[34] Zhou, P.; Zhang, J.; Zhang, Y.; Zhang, G.; Li, W.; Wei, C.; Liang, J.; Liu, Y.; Shu, S. J. Hazard. Mater. 2018, 344, 1209.

(Cheng, B.) 